\title{
The prognostic significance of lactate dehydrogenase levels in seminoma patients with advanced disease: an analysis by the Global Germ Cell Tumor Collaborative Group (G3)
}

\author{
Christoph Seidel ${ }^{1}\left[\right.$. Gedske Daugaard ${ }^{2} \cdot$ Tim Nestler $^{3} \cdot$ Alexey Tryakin $^{4} \cdot$ Mikhail Fedyanin $^{4}$. \\ Christian Daniel Fankhauser ${ }^{5}$. Thomas Hermanns $^{5}$. Jorge Aparicio ${ }^{6}$. Julia Heinzelbecker ${ }^{7} \cdot$ Pia Paffenholz $^{8,25}$. \\ Axel Heidenreich ${ }^{27}$. Ugo De Giorgi ${ }^{9} \cdot$ Richard Cathomas $^{10}$. Anja Lorch ${ }^{11,12} \cdot$ Anna Fingerhut $^{12} \cdot$ Fabian Gayer $^{13}$. \\ Felix Bremmer ${ }^{14}$. Patrizia Giannatempo ${ }^{15}$. Andrea Necchi ${ }^{15}$. Daniele Raggi ${ }^{15}$. Gaetano Aurilio ${ }^{16}$. \\ Chiara Casadei ${ }^{9}$ Marcus Hentrich ${ }^{17}$. Ben Tran ${ }^{18,26} \cdot$ Klaus-Peter Dieckmann $^{19} \cdot$ Margarido Brito $^{20} \cdot$ Christian Ruf $^{3}$. \\ Alessandro Mazzocca ${ }^{21} \cdot$ Bruno Vincenzi ${ }^{21}$. Olof Stahl ${ }^{22,23} \cdot$ Carsten Bokemeyer ${ }^{1}$. Christoph Oing ${ }^{1,24}$
}

Received: 24 November 2020 / Accepted: 5 February 2021 / Published online: 8 March 2021

(c) The Author(s) 2021

\begin{abstract}
Purpose The prognostic significance of lactate dehydrogenase (LDH) in patients with metastatic seminoma is not defined. We investigated the prognostic impact of LDH levels prior to first-line systemic treatment and other clinical characteristics in this subset of patients.

Methods Files from two registry studies and one single-institution database were analyzed retrospectively. Uni- and multivariate analyses were conducted to identify patient characteristics associated with recurrence free survival (RFS), overall survival (OS), and complete response rate (CRR).

Results The dataset included 351 metastatic seminoma patients with a median follow-up of 5.36 years. Five-year RFS, OS and CRR were $82 \%, 89 \%$ and 52\%, respectively. Explorative analysis revealed a cut-off LDH level of $<2.5$ upper limit of normal (ULN) $(n=228)$ vs. $\geq 2.5$ ULN $(n=123)$ to be associated with a significant difference concerning OS associated with 5 -years OS rates of $93 \%$ vs. $83 \%(p=0.001)$ which was confirmed in multivariate analysis (HR $2.87 ; p=0.004)$. Furthermore, the cut-off LDH $<2.5$ ULN vs. $\geq 2.5$ ULN correlated with RFS and CRR associated with a 5-years RFS rate and CRR of $76 \%$ vs. $86 \%(p=0.012)$ and $32 \%$ vs. $59 \%(p \leq 0.001)$, respectively.

Conclusions LDH levels correlate with treatment response and survival in metastatic seminoma patients and should be considered for their prognostic stratification.
\end{abstract}

Keywords Seminoma $\cdot$ Lactate dehydrogenase $\cdot$ Prognostic markers $\cdot$ Upper limit of normal

\section{Introduction}

Germ cell tumors (GCTs) are the most common solid malignancy in young men aged between 15 and 35 years [1]. Metastatic GCTs with both seminomatous and non-seminomatous histology are extremely sensitive to cisplatin-based chemotherapy as part of a multimodal treatment approach associated with 5-years overall survival (OS) rates from 50\% to $90 \%$ depending on the risk group $[2,3]$. The serum tumor

Christoph Seidel

c.seidel@uke.de

Extended author information available on the last page of the article markers beta subunit of human chorionic gonadotropin (HCG) and alpha-fetoprotein (AFP) are used for diagnosis, monitoring of treatment efficacy, and follow-up procedures [4]. In patients with non-seminomatous GCTs, the serum tumor markers HCG, AFP and LDH (lactate dehydrogenase) are used for the prognostic stratification according to the International Germ Cell Cancer Cooperation Group (IGCCCG) classification; however, tumor markers are not considered for further prognostic estimations of seminoma patients. Only approximately $30 \%$ of seminoma patients with advanced disease have serum HCG levels above normal [5-7], and unequivocally increased AFP levels are not consistent with pure seminomatous histology. In the previous work of our group, we analyzed a cohort of seminoma 
patients with elevated HCG levels and demonstrated that HCG levels are not associated with the outcome except in a small subset of advanced-stage patients with excessive HCG marker increases detected pre-orchiectomy [8]. Although pre-orchiectomy tumor markers are principally not considered for the prognostic stratification of GCT patients $[9,10]$ the results of our study revealed that HCG is not sufficient as a prognostic marker for seminoma patients [11]. Already in 1981, elevated LDH serum levels were reported to correlate with tumor bulk and impaired survival times in a small series of GCT patients [12], while other studies revealed a correlation between LDH serum levels and disease burden in GCT patients $[7,13]$. The prognostic significance of LDH levels in patients with pure seminomatous histology, however, was never yet defined. This study examines the prognostic impact of LDH levels detected prior to first-line systemic treatment concerning recurrence-free survival (RFS), overall survival (OS), and complete response rate (CRR) after forming an extended group of seminoma patients with advanced disease.

\section{Patients and methods}

\section{Study population and inclusion criteria}

We analyzed a contemporary cohort of seminoma patients who had been treated from 1983 to 2017 . The patients were retrospectively identified in 20 GCT expert centers or study groups across Europe, the Russian Federation, and Australia that participated in two previous registry studies on behalf of the global germ cell collaborative group (G3) with additional data from one single institution analysis. Previous registry studies provided data for the analysis of intermediate prognosis patients in metastatic GCT and for HCG positive seminoma patients, respectively [8, 14]. Registry studies were approved by the Ethics Committee of the Medical Association, Hamburg, Germany (Ref. No.: PV5432 and PV5050). Monocentric data acquisition was in line with local requirements according to Hamburg Hospital Act (HmbKHG)§ $12 \mathrm{HmbKHG}$. Patients were eligible for inclusion if they had (1) confirmed pure seminomatous histology according to histological examination by local pathologists, (2) advanced disease including the clinical stages IIA-C and IIIA-C according to Union for International Cancer Control (UICC) [15], (3) systemic treatment (4) availability of LDH levels detected prior to first-line systemic treatment (i.e. post-orchiectomy if carried out). Patients were excluded due to the following reasons: (1) non-seminomatous histology, (2) localized disease or (3) absence of LDH levels prior to first line treatment (4) treatment with radiotherapy only. LDH levels prior to first-line systemic treatment were determined in institutional laboratories according to international practice guidelines [16]. Due to variable measurement techniques applied in the participating institution with differences concerning reference ranges, we chose to analyze individual measurements as $\mathrm{x}$-fold values of the upper limit of normal (ULN) according to local laboratory measurement technologies.

\section{Outcome measurements and endpoint}

Our study hypothesis was that elevated LDH levels detected prior to first-line tretament inversely correlate with outcome in seminoma patients with metastatic disease. End-points of the study were, RFS defined as the time from the end of first line treatment to the date of disease recurrence, OS defined as the time from the first diagnosis until death from any cause or last date of follow-up, and complete response rate (CRR) defined as the rate of complete remission achieved during first-line chemotherapy. Complete remission was defined as no radiological evidence of residual disease and normalized tumor markers after completion of systemic treatment or radiotherapy. Patients lost to follow-up were censored at the date of their last visit.

\section{Statistical analysis}

To explore a correlation between rising LDH levels and outcome patients were stratified according to the extent of their LDH levels detected prior to first-line treatment using the following model: (1) $<1.5$ ULN vs. (2) 1.5 ULN to 2 ULN (3) vs. $>2-3$ ULN, (4) vs. $>3.0$ ULN. To find a cut-off level which reveals the highest difference between beneficial and impaired outcomes, the following cut-off levels were tested: LDH prior to first-line treatment $\geq 1.5 \mathrm{ULN}$ vs. $<1.5 \mathrm{ULN}$; $\mathrm{LDH}$ prior to first-line treatment $\geq 2.0 \mathrm{ULN}$ vs. $<2.0 \mathrm{ULN}$; LDH prior to first-line treatment $\geq 2.5 \mathrm{ULN}$ vs. $<2.5 \mathrm{ULN}$; $\mathrm{LDH}$ prior to first-line treatment $\geq 3.0 \mathrm{ULN}$ vs. $<3.0 \mathrm{ULN}$. Other potential prognostic indicators considered for statistical analysis were age, IGCCCG risk category, primary testicular vs. extragonadal, pulmonary vs. other visceral metastases, and HCG levels prior to first-line treatment $(\geq 2.000$ $\mathrm{IU} / 1$ vs. $<2.000 \mathrm{IU} / 1$ and $\geq 5.000 \mathrm{IU} / 1$ vs. $<5.000 \mathrm{IU} / 1)$. Survival analysis was conducted using the Kaplan-Meier method [17]. A log-rank test was applied to compare survival rates. Patient characteristics found to be associated 
with outcome $(p<0.1)$ were tested in a multivariate Cox regression model. Variables were found to be significant if a two-sided $p$ value was $<0.05$. To analyze the correlation between patient characteristics and CRR Pearson Chi-square test was conducted. Statistical analysis was performed using Statistical Package for the Social Sciences (SPSS, version 17.0; IBM Corp, Armonk, NY, USA).

\section{Results}

\section{Patient characteristics}

Data files from two registry studies and one single institution database were screened. Of 1183 files 351 seminoma patients with metastatic disease were considered eligible for analysis (Fig. 1). Clinical characteristics of the patient cohort are described in Table 1. The median follow-up time since diagnosis was 5.36 years (interquartile range: 6.32). The cohort included patients with clinical stages IIA-C $(n=209)$ and IIIA-C $(n=129)$ according to UICC [14]. In 13 cases the exact stage of advanced disease remained undefined. LDH levels upper limit of normal prior to first-line treatment according to local laboratory findings were detected in 261 of 351 (74\%).

\section{Treatment and response}

Systemic cisplatin-based chemotherapy according to institutional standards was administered in 274 patients (78\%). Altogether 60 systemic chemotherapy regimens were defined as 'others' (17\%) while in 13 cases further information on treatment regimens was absent (4\%). Additional surgery after systemic treatment was documented in 21 patients (6\%). Treatment response data were available in 274 patients. Complete remissions were achieved in 145 (53\%), partial remissions in 108 (39\%), stable disease in $11(4 \%)$, and progressive disease in $10(4 \%)$ patients. Details of the treatment regimens are summarized in Table 1.

\section{Correlation of patient characteristics with overall survival (OS) and recurrence-free survival (RFS)}

The 5-year OS rate of the total cohort was $89 \%$. The stratification of patients according to their LDH levels detected prior to first-line treatment suggests an inverse correlation between rising LDH levels and outcome. Five year OS rates of $94 \%$ were detected for patients with LDH levels $<1.5$ ULN ( $n=155) ; 87 \%$ for patients with LDH levels from 1.5 ULN to 2 ULN ( $n=54), 76 \%$ for patients with LDH levels from $>2$ to $3 \mathrm{ULN}(n=35)$ and $86 \%$ for patients with LDH levels $>3.0$ ULN $(n=107)(p=0.003)$ (Fig. 2). To evaluate a cut-off providing the highest discrepancy between patients with good and impaired outcomes, patients were consecutively categorized according to their extent of LDH levels. Cut-off levels tested are described in "Outcome measurements and endpoint". Regarding this stratification, our analysis revealed the cut-off level of $\mathrm{LDH} \geq 2.5 \mathrm{ULN}$ prior to firstline treatment $(n=123)$ vs. $<2.5 \mathrm{ULN}(n=228)$ providing the highest difference concerning outcome associated with a 5 -year OS rate of $83 \%$ vs. $93 \%(p=0.001)$ (Table 2; Fig. 2). Other factors correlating with an impaired OS rate were
Fig. 1 Overview of the patient cohort: total cohort divided into a number of patients in different analyses

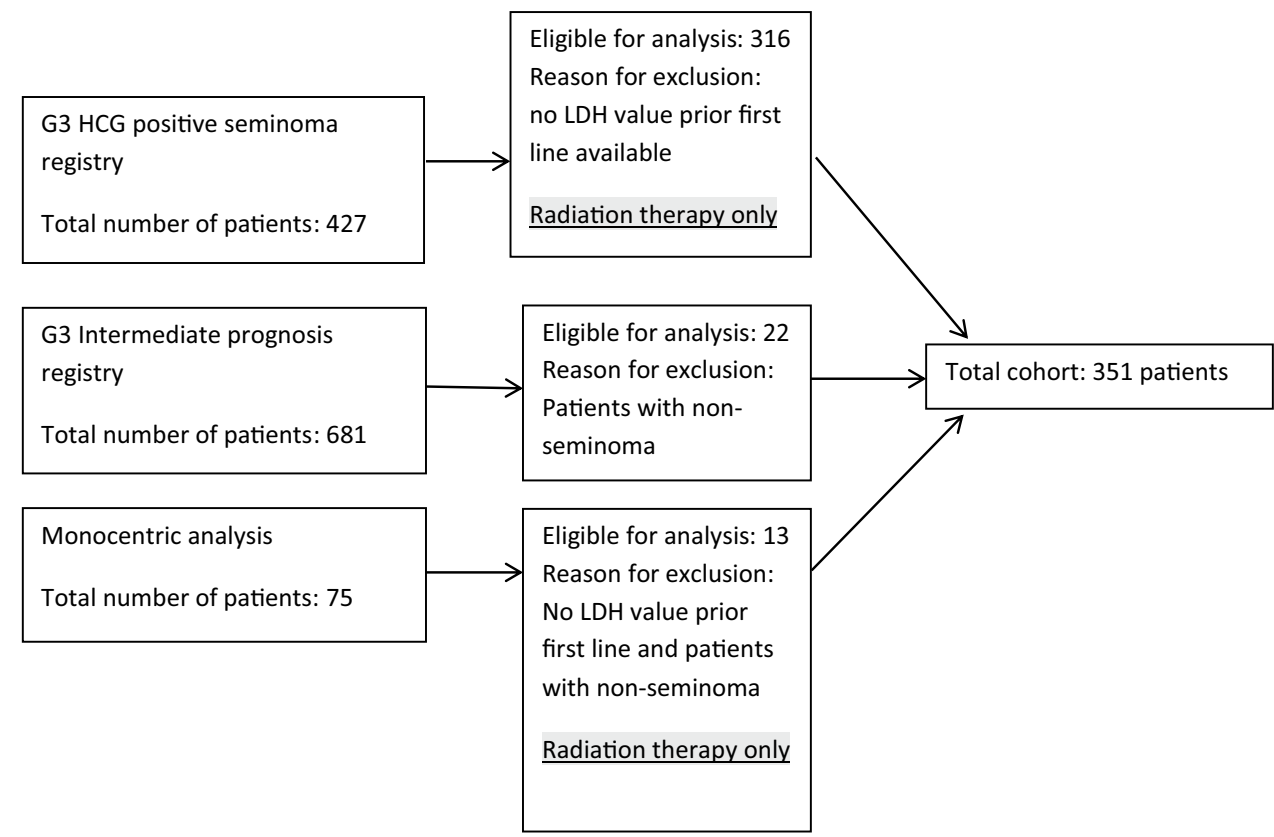


Table 1 Patient characteristics

\begin{tabular}{|c|c|c|}
\hline Characteristics & Absolute number of patients $n=351$ & $100 \%$ \\
\hline \multicolumn{3}{|c|}{ LDH values prior to first-line treatment (ULN) } \\
\hline$<1.5$ & 155 & $44 \%$ \\
\hline$>1.5-2$ & 54 & $15 \%$ \\
\hline$>2-3$ & 35 & $10 \%$ \\
\hline$>3$ & 107 & $30 \%$ \\
\hline Median age in years & 39.9 & (Range 18-79) \\
\hline \multicolumn{3}{|l|}{ Primary site of the tumor } \\
\hline Gonadal & 263 & $75 \%$ \\
\hline Extragonadal & 70 & $20 \%$ \\
\hline Missing & 18 & $5 \%$ \\
\hline \multicolumn{3}{|l|}{ IGCCCG classification } \\
\hline Good prognosis & 276 & $78 \%$ \\
\hline Intermediate prognosis & 65 & $19 \%$ \\
\hline Missing & 10 & $3 \%$ \\
\hline \multicolumn{3}{|l|}{ Stage UICC } \\
\hline UICC Stage II & 209 & $59 \%$ \\
\hline UICC Stage III & 129 & $37 \%$ \\
\hline Missing & 13 & $4 \%$ \\
\hline \multicolumn{3}{|l|}{ Treatment regimens } \\
\hline Three cycles of BEP & 126 & $36 \%$ \\
\hline Four cycles of BEP & 122 & $35 \%$ \\
\hline Three to four cycles VIP & 8 & $2 \%$ \\
\hline Four cycles of PE & 18 & $5 \%$ \\
\hline Four cycles of CE & 4 & $1 \%$ \\
\hline Defined as "others" & 60 & $17 \%$ \\
\hline Missing & 13 & $4 \%$ \\
\hline
\end{tabular}

UICC Union for International Cancer Control, BEP Bleomycin, Etoposide, Cisplatin, VIP Vindensine, Ifosfamide, Cisplatin, PE Cisplatin Etoposide, SAKK Schweizerische Arbeitsgemeinschaft für Klinische Krebsforschung

HCG levels $\geq 2.000 \mathrm{IU} / 1$ vs. $<2.000 \mathrm{IU} / 1(n=12)$ detected prior to first-line treatment associated with a 5 -year OS rate of $82 \%$ vs. $89 \%(p=0.022)$ and age above the median revealed an impaired 5-year survival rate of $86 \%$ vs. $91 \%$ vs. $(p=0.024)$. Multivariate analysis confirmed LDH $\geq 2.5 \mathrm{ULN}$ as the only independent prognostic factor concerning OS (HR 2.86; 95\% CI 1.41-5.85; $p=0.004$ ). Results of the outcome analysis are illustrated in detail in Table 2. Concerning the RFS, LDH cut-off levels and other patient characteristics were tested as well. The 5-year RFS rate was $83 \%$. Univariate analysis revealed LDH levels prior to first-line treatment as the only variable to be associated with the RFS. Here LDH levels $\geq 2.5$ ULN vs. $<2.5$ ULN were associated with a five-year RFS rate of $76 \%$ vs. $86 \%(p=0.012)$ (Table 2 ; Fig. 2). As no other variable was detected to be significant, further multivariate analysis was not conducted.

\section{Correlation of patient characteristics with complete remission rate}

The complete remission rate was $53 \%$. Chi-square test revealed $\mathrm{LDH}$ levels prior to first-line treatment to inversely correlate with CRR. Here LDH levels $\geq 2.5$ ULN vs. $<2.5$ ULN were associated with a CRR of $32 \%$ compared to $59 \%$ $(p<0.001)$, respectively. None of the other clinical characteristics significantly correlated with CRR (Table 2).

\section{Discussion}

This registry study proved a significant correlation of serum LDH levels with OS, RFS and CRR in patients with metastasized seminoma. Elevated LDH levels were already associated with poorer outcomes including several solid 
A

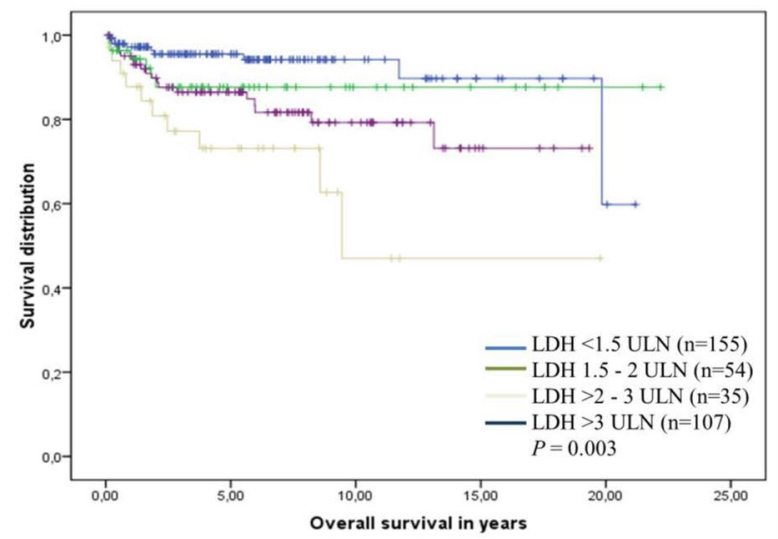

B

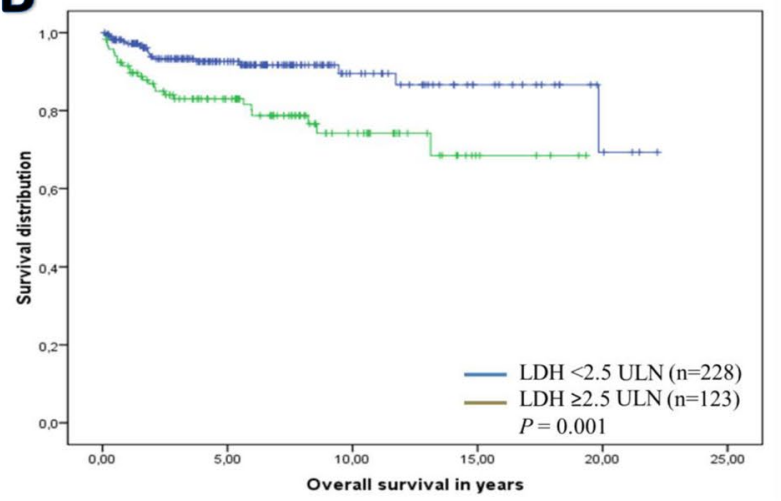

C

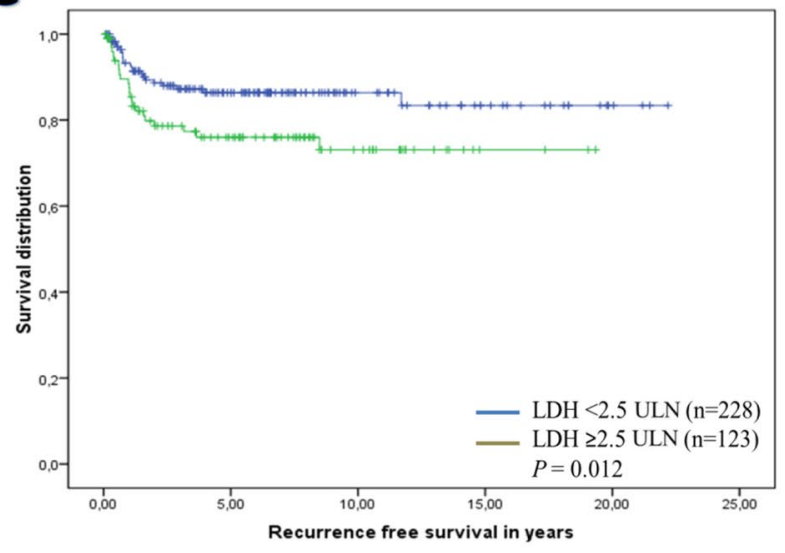

Fig. 2 Patients were stratified into four cohorts according to increasing LDH levels detected prior to first-line treatment: $<1.5$ upper limit of normal (ULN); 1.5-2; from $>2$ to 3, and $>3$ ULN. The graph demonstrates a correlation between rising LDH levels and impaired outcome concerning OS (a). Stratification of patients according to prior to first-line treatment LDH levels identifies $\geq 2.5$ ULN as a cut-off level with significant prognostic impact concerning OS (b) and RFS (c) tumor and hematologic malignancies [18-22] but the value of $\mathrm{LDH}$ as a prognostic marker for seminoma patients remained unclear. To find new categories that significantly correlate with outcome, our analysis investigates LDH levels and other patient characteristics as potential prognostic markers, hypothesizing that LDH inversely correlates with outcome in seminoma patients as well. We herein found a negative correlation between rising LDH levels and outcome concerning OS in consideration of uni- and multivariate analyses. According to different LDH thresholds, the cut-off level of $\geq 2.5$ ULN vs. $<2.5$ ULN provided a high discrepancy between patients with impaired and beneficial outcomes. Of note, this cut-off was also significantly relevant concerning RFS and CRR within our analysis and is in line with results recently reported by the EORTC IGCCCG-update consortium [23].

This analysis can add substantially new information to the field of testis cancer by identifying LDH levels $>2.5$ ULN as a novel marker to define seminoma patients at high risk.

Limitations of the study are a retrospective design and partially incomplete datasets. Another limitation is that the majority of patients were identified from an earlier registry study including exclusively seminoma patients with HCG levels above normal reference ranges. As our previous study demonstrated that HCG levels are not associated with the outcome except in a small subset of advanced-stage patients with excessive HCG marker increases detected pre-orchiectomy we think that this large subset of marker positive HCG levels in our cohort will not interfere with data of seminoma patients without any elevated HCG levels. We therefore consider the value of elevated LDH levels to identify seminoma patients with an inferior prognosis as promising new information, representing an easily applicable assessment for the daily clinical routine. Here strategies for treatment escalation or de-escalation based on LDH might be worth to be considered for future projects.

\section{Conclusions}

This retrospective series of advanced seminoma patients highlights the prognostic impact of LDH levels detected prior to first-line systemic treatment concerning OS, RFS and CRR.

LDH levels expound a distinct utility to characterize a subgroup of seminoma patients with an inferior outcome.

Additional research to further evaluate the potential utility of this marker for prognostic stratification and treatment adjustments is needed. 
Table 2 Results of univariate and multivariate analyses of OS, RFS and CRR

\begin{tabular}{|c|c|c|c|}
\hline Factor & & Difference 5-year OS rate & $\begin{array}{l}\text { Log-rank test } \\
p \text { value }\end{array}$ \\
\hline LDH prior to first-line $<1.5$ ULN vs. $\geq 1.5 \mathrm{ULN}$ & & $85 \%$ vs. $94 \%$ & 0.021 \\
\hline LDH prior to first-line $\geq 2.5$ ULN vs. $<2.0 \mathrm{ULN}$ & & $84 \%$ vs. $94 \%$ & 0.003 \\
\hline LDH prior to first-line $\geq 2.5$ ULN vs. $<2.5$ ULN & & $83 \%$ vs. $93 \%$ & 0.001 \\
\hline $\mathrm{LDH}$ prior to first-line $\geq 3.0 \mathrm{ULN}$ vs. $<3.0 \mathrm{ULN}$ & & $87 \%$ vs. $91 \%$ & 0.054 \\
\hline Age above vs. below median & & $86 \%$ vs. $91 \%$ & 0.024 \\
\hline IGCCCG good vs. intermediate & & $88 \%$ vs. $90 \%$ & 0.240 \\
\hline HCG prior to first line $>2000 \mathrm{U} / 1$ & & $82 \%$ vs. $89 \%$ & 0.022 \\
\hline HCG prior to first line $>5000 \mathrm{U} / 1$ & & $87 \%$ vs. $89 \%$ & 0.063 \\
\hline Factor & HR & $95 \% \mathrm{CI}$ & $p$ value \\
\hline LDH prior to first-line $\geq 2.5$ ULN vs. $<2.5$ ULN & 2.87 & $1.41-5.85$ & 0.004 \\
\hline HCG prior to first-line $>2000 \mathrm{U} / 1$ & 0.41 & $0.14-1.19$ & 0.102 \\
\hline Age above vs. below median & 1.67 & $0.86-3.25$ & 0.132 \\
\hline Factor & & Difference 5-year RFS rate & $\begin{array}{l}\text { Log-rank } \\
\text { test } p \\
\text { value }\end{array}$ \\
\hline $\mathrm{LDH}$ prior to first-line $\geq 1.5 \mathrm{ULN}$ vs. $<1.5 \mathrm{ULN}$ & & $79 \%$ vs. $89 \%$ & 0.027 \\
\hline $\mathrm{LDH}$ prior to first-line $\geq 2.0 \mathrm{ULN}$ vs. $<2.0 \mathrm{ULN}$ & & $78 \%$ vs. $86 \%$ & 0.082 \\
\hline LDH prior to first-line $\geq 2.5$ ULN vs. $<2.5$ ULN & & $76 \%$ vs. $86 \%$ & 0.012 \\
\hline LDH prior to first-line $\geq 3.0 \mathrm{ULN}$ vs. $<3.0 \mathrm{ULN}$ & & $78 \%$ vs. $86 \%$ & 0.087 \\
\hline Age above vs. below median & & $81 \%$ vs. $83 \%$ & 0.673 \\
\hline IGCCCG good vs. intermediate & & $82 \%$ vs. $84 \%$ & 0.933 \\
\hline HCG prior to first-line $>2000 \mathrm{U} / 1$ & & $73 \%$ vs. $84 \%$ & 0.552 \\
\hline HCG prior to first-line $>5000 \mathrm{U} / 1$ & & $72 \%$ vs. $82 \%$ & 0.063 \\
\hline Factor & & Difference CRR & $\begin{array}{l}\text { Chi-square test } \\
p \text { value }\end{array}$ \\
\hline LDH prior to first-line $\geq 1.5$ ULN vs. $<1.5 \mathrm{ULN}$ & & $39 \%$ vs. $64 \%$ & 0.005 \\
\hline LDH prior to first-line $\geq 2.0$ ULN vs. $<2.0 \mathrm{ULN}$ & & $48 \%$ vs. $50 \%$ & $<0.001$ \\
\hline LDH prior to first-line $\geq 2.5$ ULN vs. $<2.5$ ULN & & $32 \%$ vs. $59 \%$ & $<0.001$ \\
\hline $\mathrm{LDH}$ prior to first-line $\geq 3.0 \mathrm{ULN}$ vs. $<3.0 \mathrm{ULN}$ & & $21 \%$ vs. $78 \%$ & $<0.001$ \\
\hline Age $\geq$ median vs. $<$ median & & $49 \%$ vs. $52 \%$ & 0.655 \\
\hline Gonadal vs. extragonadal & & $78 \%$ vs. $22 \%$ & 0.480 \\
\hline IGCCCG good vs. intermediate & & $46 \%$ vs. $54 \%$ & 0.382 \\
\hline HCG prior to first-line $<$ vs. $>2000 \mathrm{U} / 1$ & & $53 \%$ vs. $57 \%$ & 0.821 \\
\hline HCG prior to first-line $<$ vs. $>5000 \mathrm{U} / 1$ & & $52 \%$ vs. $80 \%$ & 0.221 \\
\hline
\end{tabular}

$L D H$ lactate dehydrogenase, IGCCCG International Germ Cell Cancer Collaborative Group

Author contributions CS: conceived and designed the work that led to the submission. Drafted and revised the manuscript, acquired data, and approved the final version. GD: acquired data and approved the final version. TN: acquired data and approved the final version. AT: acquired data and approved the final version. MF: acquired data and approved the final version. CDF: acquired data and approved the final version. TH revised the manuscript and approved the final version. JA: acquired data and approved the final version. $\mathrm{JH}$ : acquired data and approved the final version. PP: acquired data and approved the final version. $\mathrm{AH}$ : revised the manuscript, played an important role in interpreting the results, and approved the final version. UDG: acquired data, revised the manuscript, and approved the final version. RC: acquired data, revised the manuscript, and approved the final version. AL: acquired data, revised the manuscript, and approved the final version. AF: acquired data and approved the final version. FG: acquired data and approved the final version. FB: acquired data and approved the final version. PG: acquired data and approved the final version. AN: acquired data and approved the final version. DR: acquired data and approved the final version. GA: acquired data, revised the manuscript, and approved the final version. $\mathrm{CC}$ : acquired data and approved the final version. $\mathrm{MH}$ : acquired data, revised the manuscript, and approved the final version. BT: acquired data, revised the manuscript, and approved the final version. KPD: approved the final version, played an important role in interpreting the results, and approved the final version. MB: acquired data and approved the final version. CR: acquired data and approved the final version. AM: acquired data and approved the final version. 
BV: acquired data and approved the final version. OS: acquired data, and approved the final version. CB: revised the manuscript, played an important role in interpreting the results, and approved the final version. CO: revised the manuscript, played an important role in interpreting the results, and approved the final version.

Funding Open Access funding enabled and organized by Projekt DEAL; F.B. is supported by the Wilhelm-Sander-Stiftung (Grant number 2016.041.1 and 2016.041.2)

\section{Declarations}

Conflict of interest The authors declare no conflict of interest.

Ethical approval This article does not contain any studies with human participants or animals performed by any of the authors.

Open Access This article is licensed under a Creative Commons Attribution 4.0 International License, which permits use, sharing, adaptation, distribution and reproduction in any medium or format, as long as you give appropriate credit to the original author(s) and the source, provide a link to the Creative Commons licence, and indicate if changes were made. The images or other third party material in this article are included in the article's Creative Commons licence, unless indicated otherwise in a credit line to the material. If material is not included in the article's Creative Commons licence and your intended use is not permitted by statutory regulation or exceeds the permitted use, you will need to obtain permission directly from the copyright holder. To view a copy of this licence, visit http://creativecommons.org/licenses/by/4.0/.

\section{References}

1. Gurney JK, Florio AA, Znaor A et al (2019) International trends in the incidence of testicular cancer: lessons from 35 years and 41 countries. Eur Urol 76:615-623. https://doi.org/10.1016/j.eurur o.2019.07.002

2. Hanna NH, Einhorn LH (2014) Testicular cancer-discoveries and updates. New Engl J Med 371:2005-2016

3. IGCCCG (1997) International Germ Cell Consensus Classification: a prognostic factor-based staging system for metastatic germ cell cancers. International Germ Cell Cancer Collaborative Group. J Clin Oncol 15:594-603

4. Gilligan TD, Seidenfeld J, Basch EM et al (2010) American Society of clinical oncology clinical practice guideline on uses of serum tumor markers in adult males with germ cell tumors. J Clin Oncol 28:3388-3404

5. Weissbach L, Bussar-Maatz R, Mann K et al (1997) The value of tumor markers in testicular seminomas. Results of a prospective multicenter study. Eur Urol 32:16-22

6. Weissbach L, Bussar-Maatz R, Löhrs U et al (1999) Prognostic factors in seminomas with special respect to HCG: results of a prospective multicenter study. Seminoma Study Group. Eur Urol 36:601

7. Dieckmann KP, Simonsen-Richter H, Kulejewski M et al (2019) Serum tumour markers in testicular germ cell tumours: frequencies of elevated levels and extents of marker elevation are significantly associated with clinical parameters and with response to treatment. BioMed Res Int. https://doi.org/10.1155/2019/50303 49 (PMID: 31275973)

8. Seidel C, Daugaard G, Nestler T et al (2020) Human chorionic gonadotropin-positive seminoma patients: a registry compiled by the global germ cell tumor collaborative group (G3). Eur J Cancer.
29:127-135. https://doi.org/10.1016/j.ejca.2020.03.022 ([Epub ahead of print])

9. Honecker F, Aparicio J, Berney D et al (2018) ESMO consensus conference on testicular germ cell cancer: diagnosis, treatment and follow-up. Ann Oncol 29:1658-1686. https://doi.org/10.1093/ annonc/mdy 217

10. Fankhauser CD, Gerke TA, Roth L et al (2019) Pre-orchiectomy tumor marker levels should not be used for International Germ Cell Consensus Classification (IGCCCG) risk group assignment. J Cancer Res Clin Oncol 145:781-785. https://doi.org/10.1007/ s00432-019-02844-z

11. Seidel C, Daugaard G, Nestler T et al (2020) Prognostic impact of LDH and HCG levels in marker-positive seminomas. J Clin Oncol 38(6_suppl):392-392. https://doi.org/10.1200/JCO.2020.38.6_ suppl.392

12. Lippert MC, Javadpour N (1981) Lactic dehydrogenase in the monitoring and prognosis of testicular cancer. Cancer 48:2274-2278

13. Taylor RE, Duncan W, Horn DB (1986) Lactate dehydrogenase as a marker for testicular germ-cell tumours. Eur J Cancer Clin Oncol 22:647-653

14. Seidel C, Daugaard G, Tryakin A, Necchi A, Cohn Cedermark G, Ståhl O, Hentrich M, Brito M, Albany C, Taza F, Gerl A, Oechsle K, Bokemeyer C (2018) Intermediate prognosis in metastatic germ cell tumours-outcome and prognostic factors. Eur J Cancer 94:16-25. https://doi.org/10.1016/j.ejca.2018.01.113

15. Sobin LH, Gospodarowicz MK, Wittekind C (2009) TNM classification of malignant tumors. UICC International Union Against Cancer, 7th edn. Wiley-Blackwell, Hoboken

16. Sturgeon CM, Duffy MJ, Stenman et al (2008) National Academy of Clinical Biochemistry laboratory medicine practice guidelines for use of tumor markers in testicular, prostate, colorectal, breast, and ovarian cancers. Clin Chem 54:e11-79

17. Kaplan EL, Meier P (1958) Nonparametric estimation from incomplete observations. J Amer Statist Assoc 53:457-481. https ://doi.org/10.2307/2281868.JSTOR2281868

18. Girgis H, Masui O, White NM et al (2014) Lactate dehydrogenase $\mathrm{A}$ is a potential prognostic marker in clear cell renal cell carcinoma. Mol Cancer 13:101. https://doi. org/10.1186/1476-4598-13-101

19. Gan J, Wang W, Yang $Z$ et al (2018) Prognostic value of pretreatment serumlactate dehydrogenase level in pancreatic cancerpatients: a meta-analysis of 18 observational studies. Medicine (Baltimore) 97:e13151. https://doi.org/10.1097/MD.0000000000 013151

20. Liu R, Cao J, Gao X, Zhang J et al (2016) Overall survival of cancer patients with serum lactate dehydrogenase greater than 1000 IU/L. Tumour Biol 37:14083-14088

21. International Non-Hodgkin's Lymphoma Prognostic Factors Project (1993) A predictive model for aggressive non-Hodgkin's lymphoma. N Engl J Med 329:987-994. https://doi.org/10.1056/ NEJM199309303291402

22. Kalaycio M, Rybicki L, Pohlman B et al (2007) Elevated lactate dehydrogenase is an adverse predictor of outcome in HLAmatched sibling bone marrow transplant for acute myelogenous leukemia. Bone Marrow Transplant 40:753-758

23. Beyer J, Collette L, Daugaard G et al (2020) Prognostic factors in advanced seminoma: an analysis from the IGCCCG Update Consortium. J Clin Oncol 38(6_suppl):386-386. https://doi. org/10.1200/JCO.2020.38.6_suppl.386

Publisher's Note Springer Nature remains neutral with regard to jurisdictional claims in published maps and institutional affiliations. 


\section{Authors and Affiliations}

\section{Christoph Seidel ${ }^{1}$ D $\cdot$ Gedske Daugaard $^{2} \cdot$ Tim Nestler $^{3} \cdot$ Alexey Tryakin $^{4} \cdot$ Mikhail Fedyanin $^{4}$.}

Christian Daniel Fankhauser ${ }^{5}$. Thomas Hermanns $^{5}$. Jorge Aparicio ${ }^{6}$. Julia Heinzelbecker ${ }^{7}$. Pia Paffenholz ${ }^{8,25}$. Axel Heidenreich ${ }^{27}$. Ugo De Giorgi ${ }^{9} \cdot$ Richard Cathomas $^{10}$. Anja Lorch ${ }^{11,12} \cdot$ Anna Fingerhut $^{12} \cdot$ Fabian Gayer $^{13}$. Felix Bremmer ${ }^{14}$. Patrizia Giannatempo ${ }^{15}$. Andrea Necchi ${ }^{15}$. Daniele Raggi ${ }^{15}$. Gaetano Aurilio ${ }^{16}$.

Chiara Casadei $^{9} \cdot$ Marcus Hentrich $^{17}$. Ben Tran ${ }^{18,26}$. Klaus-Peter Dieckmann ${ }^{19}$. Margarido Brito ${ }^{20}$. Christian Ruf ${ }^{3}$. Alessandro Mazzocca ${ }^{21}$ - Bruno Vincenzi ${ }^{21}$. Olof Stahl ${ }^{22,23} \cdot$ Carsten Bokemeyer ${ }^{1}$. Christoph Oing ${ }^{1,24}$

1 Department of Oncology, Hematology and Bone Marrow Transplantation with Division of Pneumology, University Medical Center Hamburg-Eppendorf, Martinistraße 52, 20246 Hamburg, Germany

2 Department of Oncology, Copenhagen University Hospital, Rigshospitalet, Copenhagen, Denmark

3 Department of Urology, Federal Armed Services Hospital Koblenz, Koblenz, Germany

4 Department of Clinical Pharmacology and Chemotherapy, N. N. Blokhin Russian Cancer Research Center, Moscow, Russian Federation

5 University Hospital Zürich, Zurich, Switzerland

6 Medical Oncology Department, Hospital La Fe - On behalf of the Spanish Germ Cell Cancer Group, Valencia, Spain

7 Department of Urology, University Hospital Saarland, Homburg/Saar, Germany

8 Department of Urology, University Hospital Cologne, Cologne, Germany

9 Department of Medical Oncology, Istituto Scientifico Romagnolo per lo Studio e la Cura Dei Tumori (IRST) IRCCS - On behalf of the Italian Germ Cell Cancer Group (IGG), Meldola, Italy

10 Department of Oncology/Hematology, Kantonsspital Graubünden, Chur, Switzerland

11 Department of Oncology and Hematology, University Hospital Zürich, Zurich, Switzerland

12 Department of Urology, University Hospital Düsseldorf, Düsseldorf, Germany

13 Department of Urology, University Clinic Göttingen, Göttingen, Germany
14 Department of Pathology, University Clinic Göttingen, Göttingen, Germany

15 Fondazione IRCCS, Istituto Nazionale dei Tumori, Milan, Italy

16 Medical Division of Urogenital and Head and Neck Cancer, IEO European Institute of Oncology IRCCS, Milan, Italy

17 Department of Hematology and Oncology, Red Cross Hospital, University of Munich, Munich, Germany

18 Peter MacCallum Cancer Centre, Melbourne, Australia

19 Asklepios Klinik Altona, Hodentumorzentrum, Hamburg, Germany

20 Instituto Português de Oncologia de Lisboa, Lisboa, Portugal

21 University Campus Bio-Medico, Rome, Italy

22 SWENOTECA, Trondheim, Norway

23 Department of Oncology, Skane University Hospital, Lund, Sweden

24 Mildred Scheel Career Center HaTriCS4, University Medical Center Eppendorf, Hamburg, Germany

25 Department of Urology, Medical University Vienna, Vienna, Austria

26 Walter and Eliza Hall Institute of Medical Research, Melbourne, Australia

27 Department of Urology, Uro-Onology, Robot-Assisted and Specialized Urologic Surgery, University of Cologne, Cologne, Germany 